\title{
Two-stage particle separation channel based on standing wave surface acoustic wave
}

\section{Xueye Chen ( $\nabla$ xueye_chen@126.com )}

Ludong University https://orcid.org/0000-0002-2377-352X

\section{Yaolong Zhang}

Liaoning University of Technology

\section{Research}

Keywords: Microfluidic technology, Ultrasonic standing wave, Two-stage particle separation

Posted Date: May 3rd, 2021

DOI: https://doi.org/10.21203/rs.3.rs-376194/v1

License: (a) (i) This work is licensed under a Creative Commons Attribution 4.0 International License. Read Full License 


\section{Abstract}

Microfluidic technology has great advantages in the precise manipulation of micro and nano particles, and the collection method of micro and nano particles based on ultrasonic standing waves has attracted much attention for its high efficiency and simplicity of structure. This paper proposes a two-stage particle separation channel using ultrasound. In the microfluidic channel, two different sound pressure regions are used to achieve the separation of particles with positive acoustic contrast factors. Through numerical simulation, the performance of three common piezoelectric substrate materials was compared qualitatively and quantitatively, and it was found that the output sound pressure intensity of $128^{\circ} \mathrm{YX}$ $\mathrm{LiNbO3}$ was high and the output was stable. At the same time, the influence of the number of electrode pairs of the interdigital transducer and the electrode voltage on the output sound wave is studied. Finally, 15 pairs of electrode pairs are selected, and the electrode voltages of the two sound pressure regions are $2.0 \mathrm{~V}$ and 3.0V respectively. After selecting the corresponding parameters, the separation process was numerically simulated, and the separation of three kinds of particles was successfully achieved. This work has laid a certain theoretical foundation for rapid disease diagnosis and real-time monitoring of the environment in practical applications.

\section{Introduction}

In recent years, the development of microfluidic technology in the fields of biology, chemistry, materials science, medicine and other fields has been particularly rapid. It has gradually shown a trend to replace the traditional methods of various functions realized in the above fields, involving cell sorting[1], reaction [2], mixing [3], cell location and culture [4] and many other multi-level applications[5]. In particular, a variety of new coronary pneumonia detection methods and vaccine development technologies developed by domestic and foreign researchers based on microfluidic technology have played a key role in the fight against COVID-19 this year[ 6].

With the rapid development of microfluidic technology, it has gradually begun to replace the functions of traditional conventional biochemical medical laboratories and has broad application prospects [710].Among the many applications of microfluidic technology, the sorting function is one of the most core functions. Efficient sorting is of great significance to the fields of biology, chemistry, diagnostics, therapeutics, materials synthesis and drug development $[11,12]$. The sorting method using microfluidics can be divided into active method and passive method. The passive method uses the force exerted by the particles when they flow in the microchannel to realize the sorting without applying external force to the particles. There are mainly inertial force sorting [13], hydrodynamic sorting [14], micro-structure filtering and screening [15] and other technologies. The other is an active sorting method, which is to apply an external force field to make different suspended particles in the micro flow channel move along different trajectories to achieve the purpose of sorting, mainly including dielectrophoresis sorting [16], sound force sorting $[17,18]$, fluorescence excitation sorting [19] and magnetic sorting [20], etc. These methods other than acoustic sorting are limited in practical applications due to their damage to biological particles or 
manipulation, only the particles and the medium are required to have a difference in their density or compressibility properties. The realization conditions are relatively easy, and the separation efficiency is extremely high, thus obtaining a wider range of applications.

The cells or particles in the fluid medium will be exposed to the acoustic radiation force when exposed to the sound field [21], and the sound force depends on the size of the cells or particles and its acoustic contrast factor $(\Phi)$. The differences in the physical properties of various cells lead to differences in the size and sign of their acoustic contrast factors, so that the acoustic radiation force can be used to separate and classify cells according to their mechanical properties. When there is an acoustic standing wave, cells or particles with positive and negative acoustic contrast factors will move to the pressure node and pressure antinode, respectively. The use of acoustophoresis to separate particles with opposite contrast factors has been widely studied. Filip Petersson et al. [22] successfully realized the continuous separation of lipid particles and red blood cells using standing wave ultrasound and microchannel laminar flow. They used a microchannel with a width of $350 \mu \mathrm{m}$ and a depth of $125 \mu \mathrm{m}$, and it operated in a half-wavelength standing wave mode with a pressure node in the center of the channel. Since the acoustic contrast factor of the red blood cells is negative and the lipid particles are positive, the red blood cells are collected in the middle of the channel, and finally flow out from the middle channel opening and the fat particles are collected to the position of the channel wall, and finally flow out from the two side channel openings. Carl Grenvall et al. [23] improved the separation device to achieve the separation of somatic cells and fat particles in milk. They added two target flow channels at the entrance of the channel, and avoided the problem of particle adhesion and blockage by changing the number of nodes in the channel.

However, most cells show a positive acoustic contrast factor in an aqueous solution. In the standing wave sound field, these cells will move to the pressure node. Therefore, the use of cell diameter or the size of the acoustic contrast factor to perform cell separation methods with the same acoustic contrast factor sign has attracted widespread attention. Petersson et al. [24] proposed a microfluidic device for sorting based on cell size, which achieved the sorting of red blood cells, platelets and white blood cells by using acoustic methods. There is a pressure node in the center of the microchannel. They use the target flow to focus the cell flow on both sides of the channel. The cells begin to migrate to the pressure node due to the acoustic radiation force. Because the three kinds of cells receive different acoustic radiation forces, In the same time, the distance to the node is different, and the cells that experience different acoustic radiation force are separated and collected at different positions of the exit. Tony Jun Huang et al. [25] designed a new structure to sort white blood cells. They tilted two pairs of interdigital transducers with the flow channel at a certain angle, so that the particles have a greater migration distance, which solves the problem that the migration distance of ordinary structures is limited by the distance between pressure nodes when sorting. Augustsson et al. [26] used two acoustic standing waves to separate prostate cancer cells and white blood cells. They used two sets of standing waves at the upstream and downstream of the microchannel, the first set of standing waves focused the cells on both sides of the flow channel wall, and the second set of standing waves made the cells migrate to the central node. Two types of cells with Loading [MathJax]/jax/output/CommonHTML/jax.js ent outlets. Harris et al. [27] proposed using three acoustic 
standing waves with frequency switches to separate cells. Using the different speeds of the cells to pressure nodes at different positions at different frequencies, by switching the different acoustic field resonance modes of the channel to separate cells of different sizes or contrast factors, this design greatly reduces the restriction on the residence time of the target cells in ordinary devices .

In this article, we propose a two-stage particle separation channel based on standing wave surface acoustic waves (SSAW) to separate three kinds of particles with positive acoustic contrast factors in microfluidic channels. The fluid dynamics focusing technology is used to control the initial position of the particles, and the separation of the particles is achieved through two different sound pressure regions. The first section is the low pressure zone, which is used to separate $9.4 \mu \mathrm{m}$ particles, and the second section is the high pressure zone, which is used to separate the remaining two particles with a smaller volume difference.

This paper studies in detail the process of particle separation using acoustic surface standing wave devices. First, we selected piezoelectric substrate materials, and compared the properties of three common piezoelectric materials qualitatively and quantitatively. Subsequently, the influence of the number of electrode pairs of the interdigital transducer and the electrode voltage on the output acoustic wave was studied. After selecting the corresponding appropriate parameters, the corresponding separation model was constructed and simulated, and the separation of three different sizes of particles was successfully achieved.

\section{Methodology}

This paper uses the finite element software COMSOL Multiphysics5.0, using surface acoustic wave devices to simulate the separation of particles in the microchannel. In order to reduce the amount of simulation calculation and ensure the accuracy of the simulation, we can adopt the following assumptions:

1. The surface acoustic wave only propagates on the surface of the substrate and shows a geometric attenuation in the thickness direction. Therefore, only 1-3 times the wavelength depth can be simulated to reflect its surface wave characteristics well;

2. The propagation direction of the surface acoustic wave is perpendicular to the direction of the acoustic aperture of the electrode, and the displacement in the direction of the acoustic aperture is not coupled to the wave equation, and the amount of field remains unchanged along the length of the electrode, so the three-dimensional structure model can be simplified into a two-dimensional structure model;

3. Ignore the gravitational influence of fluids and particles on the microscopic scale.

\subsection{Surface acoustic wave device}

Surface acoustic wave refers to the elastic wave generated on the solid surface and propagated along the Loading [MathJax]/jax/output/CommonHTML/jax.js irface acoustic wave to propagate and process the signal is 
the surface acoustic wave device, which is composed of piezoelectric material and two sets of interdigital fingers on the surface. The transducer is composed, and its basic structure is shown in Fig. 1. As this article mainly studies the application of surface acoustic wave energy, both sets of interdigital transducers will be used as input terminals.

There, $\mathrm{W}$ is the acoustic aperture of the transducer, $\mathrm{a}$ is the spacing of the fingers, $\mathrm{b}$ is the width of the fingers, and $\mathrm{M}$ is the length of the periodic section. In addition, the number of interdigital pairs $\mathrm{N}$ determines the intensity of the excitation surface acoustic wave. When $a=b=M / 4$, it is called uniform interdigital transducer, which is also the type of interdigital transducer used in this article. When the same excitation signal is applied to the interdigital electrodes at both ends, two series of SAWs with the same amplitude, the same frequency, and opposite directions will be generated. After these two waves are superimposed, a standing wave field will be generated. The particle at the node after the superposition does not vibrate with time, and the amplitude of the particle at the antinode is the largest. According to the principle of wave interference, when the following conditions are met, the intensity of the surface acoustic wave generated by the transducer excitation is the largest:

$$
\mathrm{f}=f_{0}
$$

1

$$
\lambda=\mathrm{M}=v_{S} / f_{0}
$$

2

$$
\mathrm{a}=\frac{p}{2}=\frac{M}{4}=\frac{\lambda}{4}
$$

There, $f$ is the frequency of the $A C$ signal loaded on the transducer, $f_{0}$ is the resonant frequency of the transducer, $\lambda$ is the wavelength of the acoustic wave, and $v_{s}$ is the propagation velocity of the surface acoustic wave in the substrate.

\subsection{Governing equations}

The principle of surface acoustic wave standing wave sorting is that when the surface acoustic wave standing wave acts on the fluid in the micro channel, due to the acoustic flow effect, periodic pressure nodes and pressure anti-nodes will be formed in the channel. Suspended particles will migrate to the pressure node or the pressure inverse node under the action of the acoustic radiation force. The suspended particles of different properties (density, diameter, compressibility) receive different acoustic radiation force, so the migration speed and distance are also different, and then Realize sorting. Under the action of the sound field, the expression of the acoustic radiation force acting on the particles in the fluid is: 


$$
\mathrm{F}=-\left(\frac{\Pi P_{a}^{2} V_{p} K_{0}}{2 \lambda}\right) \Phi(K, \rho) \sin (2 k x)
$$

$$
\Phi(K, \rho)=\frac{\left(5 \rho_{p}-2 \rho_{0}\right)}{\left(5 \rho_{p}+\rho_{0}\right)}-\frac{K_{p}}{K_{0}}
$$

There, $\mathrm{P}_{\mathrm{a}}$ is the peak sound pressure in the channel, $\mathrm{V}_{\mathrm{p}}$ is the particle volume, $\mathrm{K}_{\mathrm{p}}$ and $\mathrm{K}_{0}$ are the compressibility of particles and water respectively, and $\rho_{p}$ and $\rho_{0}$ are the density of particles and water respectively. $\Phi$ is the acoustic contrast factor. When $\Phi>0$, the acoustic radiation force drives the particles to move toward the pressure node; when $\Phi<0$, the acoustic radiation force drives the particles to gather toward the pressure anti-node.

In addition to the acoustic radiation force, the particles are also affected by the hydrodynamic force from the surrounding fluid. The force acting direction is orthogonal to the sound force and drives the particles out of the microchannel along the liquid flow direction. Since the fluid flow in the microchannel is laminar and the particle volume is relatively small, the hydrostatic force can be approximated by the Stokes formula:

$$
F_{\text {stokes }}=-6 \pi \eta \mathrm{r}\left(\mathrm{v}_{\mathrm{p}}-\mathrm{v}_{\mathrm{f}}\right)
$$

Where $\eta$ represents the dynamic viscosity of the fluid, $r$ represents the radius of the particle, $v_{p}$ represents the velocity of the particle, and $v_{f}$ represents the flow velocity of the flow field where the particle is located.

\subsection{Working mechanism}

Most of the cells used in the experiment have a positive acoustic contrast factor. Therefore, driven by the sound radiation force, these particles will move toward the pressure node. This article uses polystyrene microspheres to replace blood cells and simulates the separation process in the microchannel. The specific parameters are shown in Table 1. By setting two zones with different sound pressures in the microchannel, the separation of three different sizes of particles is achieved, and the specific principle is shown in Fig. 2.

Table.1 Material parameters 


\begin{tabular}{|llllll|}
\hline Material & $\begin{array}{l}\text { Density } \\
(\mathrm{kg} / \mathrm{m} 3)\end{array}$ & $\begin{array}{l}\text { Speed of } \\
\text { sound } \\
(\mathrm{m} / \mathrm{s})\end{array}$ & $\begin{array}{l}\text { Compressibility } \\
(\mathrm{T} / \mathrm{Pa})\end{array}$ & $\begin{array}{l}\text { Dynamic } \\
\text { viscosity } \\
(\mathrm{mPa} \mathrm{s})\end{array}$ & $\begin{array}{l}\text { Particle } \\
\text { diameter } \\
(\boldsymbol{\mu m})\end{array}$ \\
\hline Water & 997 & 1497 & 448 & 0.890 & \\
\hline Polystyrene & 1050 & 2350 & 249 & & $9.4,5,1.8$ \\
\hline
\end{tabular}

The device uses two different transducer voltages corresponding to two different sound pressure regions in the microchannel. The standing waves passing through the microchannel form two pressure nodes in the width direction, so that the particles move toward both sides of the channel. The size of the acoustic radiation force received by the particles is different to realize the separation of the particles. The specific process is that at the entrance of the microchannel, the target stream is used to pre-focus the particles in the center of the channel to make better use of the channel space. Next, the three particles will enter the low sound pressure zone. Since the particle with a diameter of $9.4 \mu \mathrm{m}$ (corresponding to white blood cells) is larger than the other two particles, according to the above formula of the acoustic radiation force on the particle, the larger particle will be affected The greater the sound force, the faster it moves to the nodes on both sides, so the $9.4 \mu \mathrm{m}$ particles will first reach the exits on both sides of the channel to achieve separation. However, the smaller $5.0 \mu \mathrm{m}$ particles (corresponding to red blood cells) and $1.8 \mu \mathrm{m}$ particles (corresponding to platelets) will continue to enter the high sound pressure area. In the high sound pressure zone, the $5.0 \mu \mathrm{m}$ particles will eventually flow out from the outlets on both sides of the channel due to greater acoustic radiation force, while the $1.8 \mu \mathrm{m}$ particles will flow out from the middle outlet of the channel. Finally, the three kinds of particles were successfully separated based on size by using acoustic properties.

\subsection{Simulation setup}

According to the above discussion, the study of a complete physical model based on SSAW particle separation needs to consider the influence of static electricity, elasticity and fluid dynamics on the system. It is very difficult to simulate a complete three-dimensional system model and requires considerable computational cost. At the same time, since the displacement in the direction of the acoustic aperture is not coupled to the wave equation, the amount of field remains unchanged along the length of the electrode. Therefore, we can simplify the three-dimensional structure model to a twodimensional structure model, as shown in Fig. 3.

As shown in Fig. 3, we carried out a two-dimensional modeling of the required device. On the $128^{\circ} \mathrm{YX}-$ LiNbO3 substrate, 15 pairs of interdigital electrodes are distributed on the left and right sides. The electrode material is metal aluminum, and the electrode width and spacing are both $75 \mu \mathrm{m}$, the electrode thickness is $0.2 \mu \mathrm{m}$, and the interdigital transducer period is $300 \mu \mathrm{m}$. Since the surface acoustic wave decays exponentially as the depth of the material increases, the thickness of the substrate is set to 2.5 wavelengths. The middle part of the substrate surface is a microchannel with particles separated, and its width is designed to be $160 \mu \mathrm{m}$, which is just slightly larger than half the wavelength. There are three Loading [MathJax]/jax/output/CommonHTML/jax.js 
the particle flow in the middle of the channel, and the middle is the particle inlet. After passing through the low pressure zone, $9.4 \mu \mathrm{m}$ particles will flow out from the middle outlet of the microchannel, $5.0 \mu \mathrm{m}$ particles will flow out from the oulets on both sides of the microchannel tail after passing through the high pressure zone, and $1.8 \mu \mathrm{m}$ particles will flow out from the middle outlet at the end of the microchannel. Finally, the separation of the three particles is achieved.

\section{Results And Discussion}

\subsection{Selection of piezoelectric substrate materials}

Piezoelectric material is a single crystal or polycrystalline solid material that can generate electric charge under pressure. It is an important carrier for surface acoustic wave devices to conduct electroacoustic conversion and propagate surface acoustic waves. The performance of different piezoelectric materials will affect the surface acoustic wave devices made. This paper selects three common piezoelectric substrate materials, $128^{\circ} \mathrm{YX}$-LiNbO3, ZnO single crystal and piezoelectric ceramic PZT-5H, and compares and analyzes their piezoelectric properties, so as to select a suitable substrate material.

Generally, the number of electrode pairs in interdigital transducers is relatively large. In order to reduce the amount of calculation, periodic boundary conditions can be used to further simplify the model. The simplified surface acoustic wave resonator model is shown in Fig. 4(a). The thickness and width of the piezoelectric substrate are equal to one wavelength, the electrode width is equal to a quarter of the wavelength, and the thickness is $0.2 \mu \mathrm{m}$. Since the surface acoustic wave only occurs in the 1-2 wavelength range of the substrate surface during the propagation process, the surface of the surface acoustic wave device can be divided more densely. At the same time, in order to retain the information of the surface acoustic wave within one period, one wavelength should be divided into at least 5 grids, as shown in Fig. 4(b).

After the mesh is divided, the model is analyzed with modal analysis and harmonious response analysis. The speed of surface acoustic wave in the piezoelectric substrate $128^{\circ} \mathrm{YX}$-LiNbO3, ZnO single crystal and piezoelectric ceramic PZT-5H is vs $=3990 \mathrm{~m} / \mathrm{s}$, vs $=2684 \mathrm{~m} / \mathrm{s}$ and $\mathrm{vs}=2099 \mathrm{~m} / \mathrm{s}[28-30]$, according to Eq. 2, it can be concluded that the theoretical resonance frequencies of the three materials are $13.30 \mathrm{MHz}$, 8.95MHz and 6.99MHz. Based on this, Fig. 5 shows the actual resonance frequencies of the three materials are $13.13 \mathrm{MHz}, 8.98 \mathrm{MHz}$ and $6.71 \mathrm{MHz}$. It can be seen from the Fig. 5 that when it is at the resonance frequency, the model vibration shape is a surface acoustic wave waveform, and the center node of the electrode has the largest displacement and the surface acoustic wave energy is the largest.

A two-dimensional model is established, and the central water area on the surface of the surface acoustic wave device is selected as the observation object, when the input signal frequency is the resonance frequency, the number of interdigital electrode pairs is 5 , the terminal voltage is $10 \mathrm{~V}$, and the piezoelectric substrate is the above three materials, analyzing the sound pressure distribution in the water area, as shown in Fig. 6. It can be found that when the substrate is piezoelectric ceramic PZT-5H, the sound preccure in the water is the larrect followed by $128^{\circ} \mathrm{YX}$-LiNbO3, ZnO single crystal is the smallest. Loading [MathJax]/jax/output/CommonHTML/jax.js 
However, when the substrate is $128^{\circ} \mathrm{YX}$-LiNbO3 and $\mathrm{ZnO}$ single crystal, the sound pressure distribution in the water is relatively more stable. Therefore, after comprehensive consideration, it is considered that the piezoelectric performance of $128^{\circ} \mathrm{YX}$-LiNbO3 is relatively best, and it is selected as the device substrate material.

\subsection{Selection of electrode pairs for interdigital transducer}

The number of electrode pairs of interdigital transducers is one of the main factors affecting the energy output of surface acoustic wave devices. During the propagation of surface acoustic waves on the surface of the substrate, the amplitude of the particles on the substrate surface will gradually attenuate as the propagation distance increases, and the energy will gradually be dissipated. Generally speaking, the more electrode pairs, the greater the intensity of the excited surface acoustic wave. However, too many interdigital electrodes will also affect the stability of the acoustic wave output. Therefore, it is necessary to select a reasonable number of interdigital electrode pairs.

A two-dimensional model is established as shown in Fig. 3 , and the surface center of the surface acoustic wave device is selected as the observation object. When the input signal frequency is $13.13 \mathrm{MHz}$ and the number of interdigital electrode pairs is 5 pairs, 10 pairs, 15 pairs and 20 pairs, the output waveform of the observation object is shown in Fig. 7.

It can be seen from the Fig. 7 that when the number of electrode pairs is small (the number of electrode pairs is less than 20), the output waveform is relatively stable, and the intensity of the surface acoustic wave increases as the number of electrode pairs increases. When the number of electrode pairs is 20 , the surface acoustic wave output waveform fluctuates and becomes extremely unstable, and the intensity of the acoustic wave will be reduced accordingly. The possible reason is that too many electrodes interfere with each other in addition to the superposition, and the interference has a greater impact on the sound wave, which leads to the instability and the decrease of the intensity of the sound wave. Therefore, the final selection of the number of pairs of interdigital transducers is 15 pairs.

\subsection{Selection of transducer electrode voltage}

The electrode voltage of the interdigital transducer also affects the energy output of the surface acoustic wave device. Generally speaking, the greater the voltage, the greater the amplitude of the mass pointon the substrate surface and the greater the intensity of the sound wave. It is necessary to find a suitable terminal voltage so that the microchannel can generate sound pressure conditions that meet the smooth separation of particles.

As shown in Fig. 3, the absolute value of the maximum sound pressure in the microchannel liquid is selected as the observation object. Under the condition that the frequency is $13.13 \mathrm{MHz}$ and the hard sound field boundary is set on both sides of the water area, the absolute value of the maximum sound pressure in the liquid is analyzed when the electrode voltage is increased from $1.0 \mathrm{~V}$ to $2.5 \mathrm{~V}$, and the result is shown in Fia. 8(a). It can be found that the electrode voltage has a linear relationship with the surface Loading [MathJax]/jax/output/CommonHTML/jax.js 
acoustic wave output intensity, and the acoustic output intensity will increase as the voltage increases. Through simulation analysis, the sound pressure in the low sound pressure zone of the microchannel that

meets the separation conditions is $1.7 \mathrm{e}^{5} \mathrm{~Pa}$, the sound pressure in the high sound pressure zone is $2.56 \mathrm{e}^{5}$ $\mathrm{Pa}$, and the corresponding electrode voltages are $2.0 \mathrm{~V}$ and $3.0 \mathrm{~V}$. The specific sound pressure distribution is shown in Fig. 8(b).

\subsection{The separation process of particles}

After selecting the appropriate piezoelectric substrate material, the number of interdigital transducer electrode pairs, and the electrode voltage, the specific separation process of the three particles is simulated, as shown in Fig. 9. Figu.9(a) shows the flow field distribution diagram in the microchannel. There are three entrances at the beginning of the channel, of which two sides are the sheath flow inlets, and the particle flow at the middle entrance is focused on the center of the channel. It can be found that the velocity of the flow field in the center of the microchannel is the largest, and the closer to the sidewall, the lower the velocity. At the same time, the velocity of the flow field in the first half of the microchannel is relatively large, and the velocity of the flow field in the second half of the microchannel is relatively low. The reason is that the liquid splits at the two outlets in the middle of the channel, which makes the fluid velocity drop. In addition, in order to prevent all fluid from flowing out of the middle section outlet, the corresponding boundary conditions need to be set: the fluid at the outlet is controlled by pressure, the pressure at the terminal outlet is set to atmospheric pressure, that is $\mathrm{P}=0$, and the pressure at the middle section outlet of the channel is set to $P=0.45$.

Figure 9(b) shows the specific separation process of the three kinds of particles under the combined action of the flow field and the sound field. When $t=0 \mathrm{~s}$, the particle flow is ready to enter the channel at the middle entrance of the beginning of the microchannel. At $t=0.25 \mathrm{~s}$, the particle stream is focused towards the center of the microchannel under the action of the sheath flows on both sides, and begins to enter the low sound pressure zone of the microchannel. At $t=0.85 \mathrm{~s}$, the $9.4 \mu \mathrm{m}$ particles have a larger volume difference from the other two kind of particles, so them receives a greater acoustic radiation force, and produces greater displacement to both sides during the process of passing through the low sound pressure zone, thus them flows out from the middle end of the microchannel, while the remaining two kind of particles continue to flow to the high sound pressure area of the channel. At $t=2.4 \mathrm{~s}$, under the action of the high sound pressure field, the $5.0 \mu \mathrm{m}$ particles finally flow out from the outlets on both sides of the channel terminal, while the $1.8 \mu \mathrm{m}$ particles flow out from the middle outlet due to the small displacement to both sides. Finally, the three kinds of particles were successfully separated based on size by using acoustic properties.

\section{Conclusion}

We demonstrated a device that uses two different sound pressure regions to achieve particle separation in a fluid microchannel. The separation process is completed by the combination of different intensities 
of acoustic radiation force and fluid dynamics in the two sound pressure domains. The following conclusions can be drawn from the research in this article:

1. 1. Among the three piezoelectric substrate materials $128^{\circ} \mathrm{YX}$-LiNbO3, $\mathrm{ZnO}$ single crystal and piezoelectric ceramics PZT-5H, under selected parameters, $128^{\circ} \mathrm{YX}$-LiNbO3 has relatively better pressure electrical performance, the output sound pressure intensity is large, and the output is stable.

2. 2. When the number of electrode pairs of the interdigital transducer is small, the surface acoustic wave output is stable, and the output intensity increases as the number of electrode pairs increases. However, when the number of electrode pairs is 20 , interference occurs between the electrodes, which causes the waveform of the surface acoustic wave to be unstable and the output intensity is also weakened. After a comprehensive comparison, 15 pairs of electrodes were finally selected.

3. 3. In a surface acoustic wave device, the electrode voltage has a linear relationship with the output intensity of the surface acoustic wave. The acoustic output intensity will increase with the increase of the voltage. The corresponding electrode voltage can be selected according to the needs.

4. 4. To simulate the separation process of three kinds of particles, it is the key to select appropriate parameters to satisfy various balance constraints. At the same time, corresponding boundary conditions should be set at the middle exit of the microchannel to prevent all liquid streams from flowing out of the exit.

\section{Declarations}

\section{Acknowledgment}

This work was supported by Young Taishan Scholars Program of Shandong Province of China(tsqn2020),Shandong Provincial Natural Science Foundation (ZR2021JQ).

\section{Notes}

All authors declare no conflict of interest.

\section{Data Availability}

The data that supports the findings of this study are available within the article .

\section{References}

1. Zhang Y, Chen X. Dielectrophoretic microfluidic device for separation of red blood cells and platelets: a model-based study. Journal of the Brazilian Society of Mechanical Sciences Engineering. 2020;42(2):89.

2. Rahimi M, Aghel $B$, Alitabar $M$, et al. Optimization of biodiesel production from soybean oil in a microreactor. Energy Convers Manag. 2014;79:599-605. 
3. Gidde RR. Pawar P M, Ronge BP, et al Evaluation of the mixing performance in a planar passive micromixer with circular and square mixing chambers. Microsyst Technol, 2018, 24(6): 2599-610.

4. Mehling M, and Savaş, Tay. "Microfluidic cell culture." Current opinion in Biotechnology 25 (2014): 95-102.

5. Jing D, Song J. Comparison on the hydraulic and thermal performances of two tree-like channel networks with different size constraints. Int J Heat Mass Transf. 2019;130:1070-4.

6. Kim E, et al. "Microneedle array delivered recombinant coronavirus vaccines: Immunogenicity and rapid translational development." EBioMedicine (2020): 102743.

7. Yin Z, Wang W. Structure-Induced Method for Circular Cross-Sectional Nanochannel Fabrication. J Nanosci Nanotechnol. 2019;19(9):5750-4.

8. Zhou T, Song Z, Zhang X, et al. Optimal Solvent Design for Extractive Distillation Processes: A Multiobjective Optimization-Based Hierarchical Framework. Industrial Engineering Chemistry Research. 2019;58(15):5777-86.

9. Jing D, Song J. Comparison on the hydraulic and thermal performances of two tree-like channel networks with different size constraints. Int J Heat Mass Transf. 2019;130:1070-4.

10. Cheng $E$, Zou H, Yin Z, et al. Fabrication of 2D polymer nanochannels by sidewall lithography and hot embossing. J Micromech Microeng. 2013;23(7):075022.

11. Hyung Jin Sung. High frequency travelling surface acoustic waves for microparticle separation. $J$ Mech Sci Technol. 2016):;30(9 ():3945-52. Destgeer G, Alazzam A, and.

12. Devendran C, et al "Batch process particle separation using surface acoustic waves (SAW): integration of travelling and standing SAW." RSC advances 6.7 (2016): 5856-5864.

13. Warkiani M Ebrahimi, et al. "An ultra-high-throughput spiral microfluidic biochip for the enrichment of circulating tumor cells." Analyst 139.13 (2014): 3245-3255.

14. Chiu Y-Y, Huang C-K, and Yen-Wen Lu. "Enhancement of microfluidic particle separation using crossflow filters with hydrodynamic focusing." Biomicrofluidics 10.1 (2016): $011906 .$.

15. Shen S, et al "High-throughput rare cell separation from blood samples using steric hindrance and inertial microfluidics." Lab on a Chip 14.14 (2014): 2525-2538.

16. Zhang $Y$, Chen $X$. Blood cells separation microfluidic chip based on dielectrophoretic force. Journal of the Brazilian Society of Mechanical Sciences Engineering. 2020;42:1-11.

17. Ding X, et al "Standing surface acoustic wave (SSAW) based multichannel cell sorting." Lab on a Chip 12.21 (2012): 4228-4231.

18. Shi J, et al "Continuous particle separation in a microfluidic channel via standing surface acoustic waves (SSAW)." Lab on a Chip 9.23 (2009): 3354-3359.

19. Wu T-H, et al "Pulsed laser triggered high speed microfluidic fluorescence activated cell sorter." Lab on a Chip 12.7 (2012): 1378-1383.

20. Hejazian M, Li W, and Nam-Trung N. "Lab on a chip for continuous-flow magnetic cell separation." 
21. Yosioka $\mathrm{K}$, and Kawasima Y. "Acoustic radiation pressure on a compressible sphere." Acta Acustica united with Acustica 5.3 (1955): 167-73.

22. Petersson F, et al "Continuous separation of lipid particles from erythrocytes by means of laminar flow and acoustic standing wave forces." Lab on a Chip 5.1 (2005): 20-22.

23. Grenvall. Carl, et al. "Harmonic microchip acoustophoresis: a route to online raw milk sample precondition in protein and lipid content quality control." Analytical chemistry 81.15 (2009): 6195200.

24. Petersson F, et al "Free flow acoustophoresis: microfluidic-based mode of particle and cell separation." Analytical chemistry 79.14 (2007): 5117-5123.

25. Ding $X$, et al. "Cell separation using tilted-angle standing surface acoustic waves." Proceedings of the National Academy of Sciences 111.36 (2014): 12992-12997..

26. Augustsson P, et al "Microfluidic, label-free enrichment of prostate cancer cells in blood based on acoustophoresis." Analytical chemistry 84.18 (2012): 7954-7962.

27. Harris N, et al "A novel binary particle fractionation technique Phys." Procedia 3 (2010): 277-281.

28. Wang $C$, et al "Characteristics of SAW hydrogen sensors based on InOX/128 ${ }^{\circ} \mathrm{YX}$-LiNbO3 structures at room temperature." Sensors \& Actuators B Chemical 173(2012):710-715.

29. Kobiakov IB. "Elastic, piezoelectric and dielectric properties of ZnO and CdS single crystals in a wide range of temperatures." Solid State Communications 35.3(1980):305-310.

30. Ruizhi, Wang T Enling, and Guolai Y. "Dynamic Piezoelectric Properties of PZT Under Shock Compression." Physica status solidi 216.6(2019):1800859.1-1800859.6..

\section{Figures}

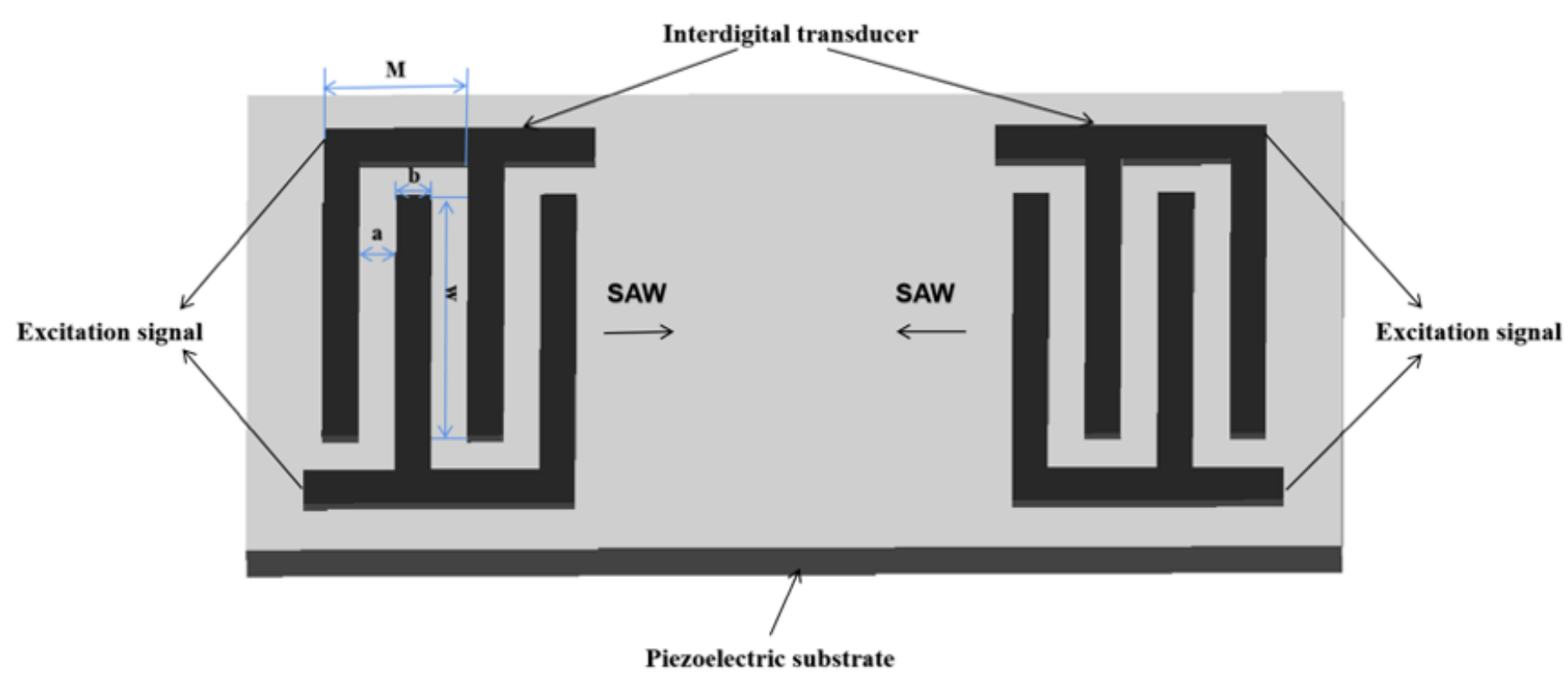

\section{Figure 1}




\section{Flow direction}

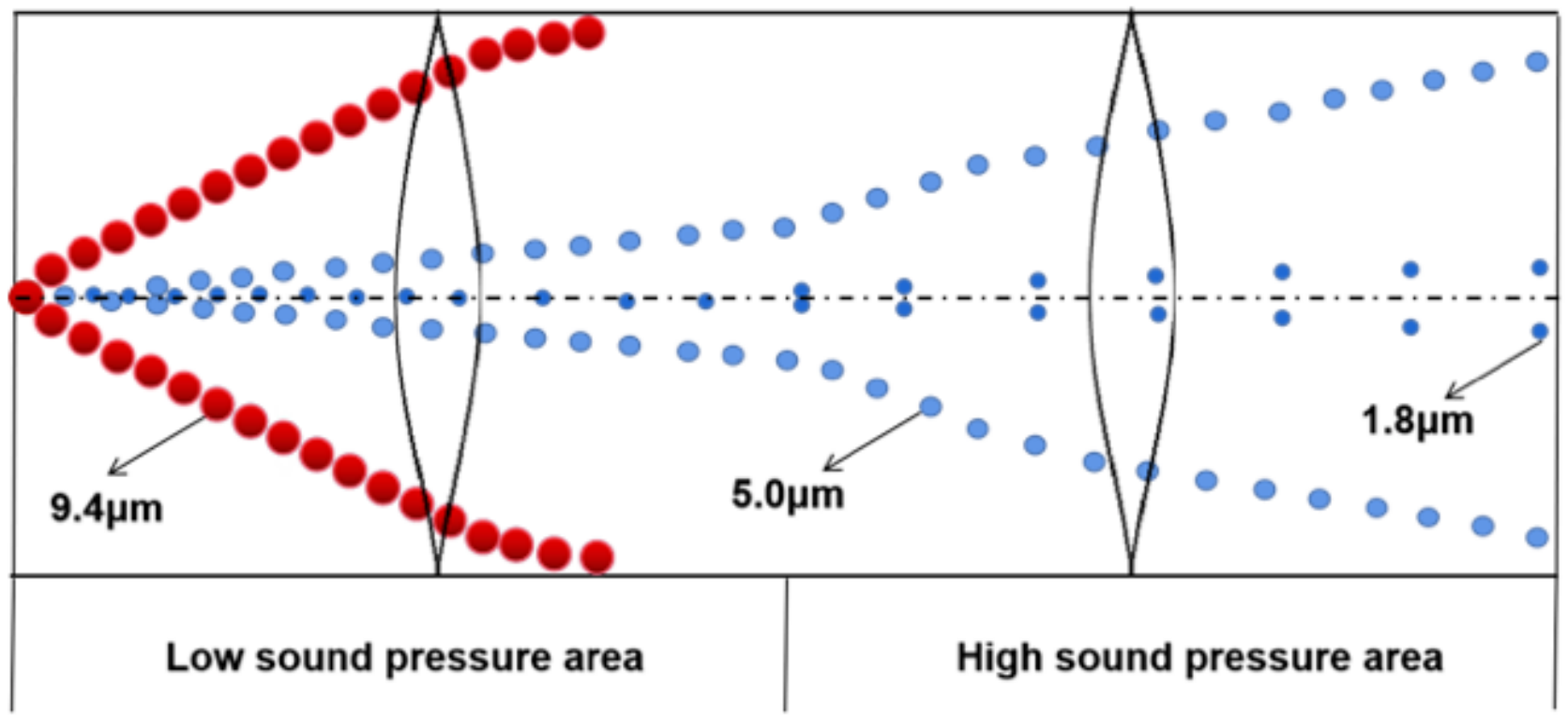

Figure 2

Principle of particle separation

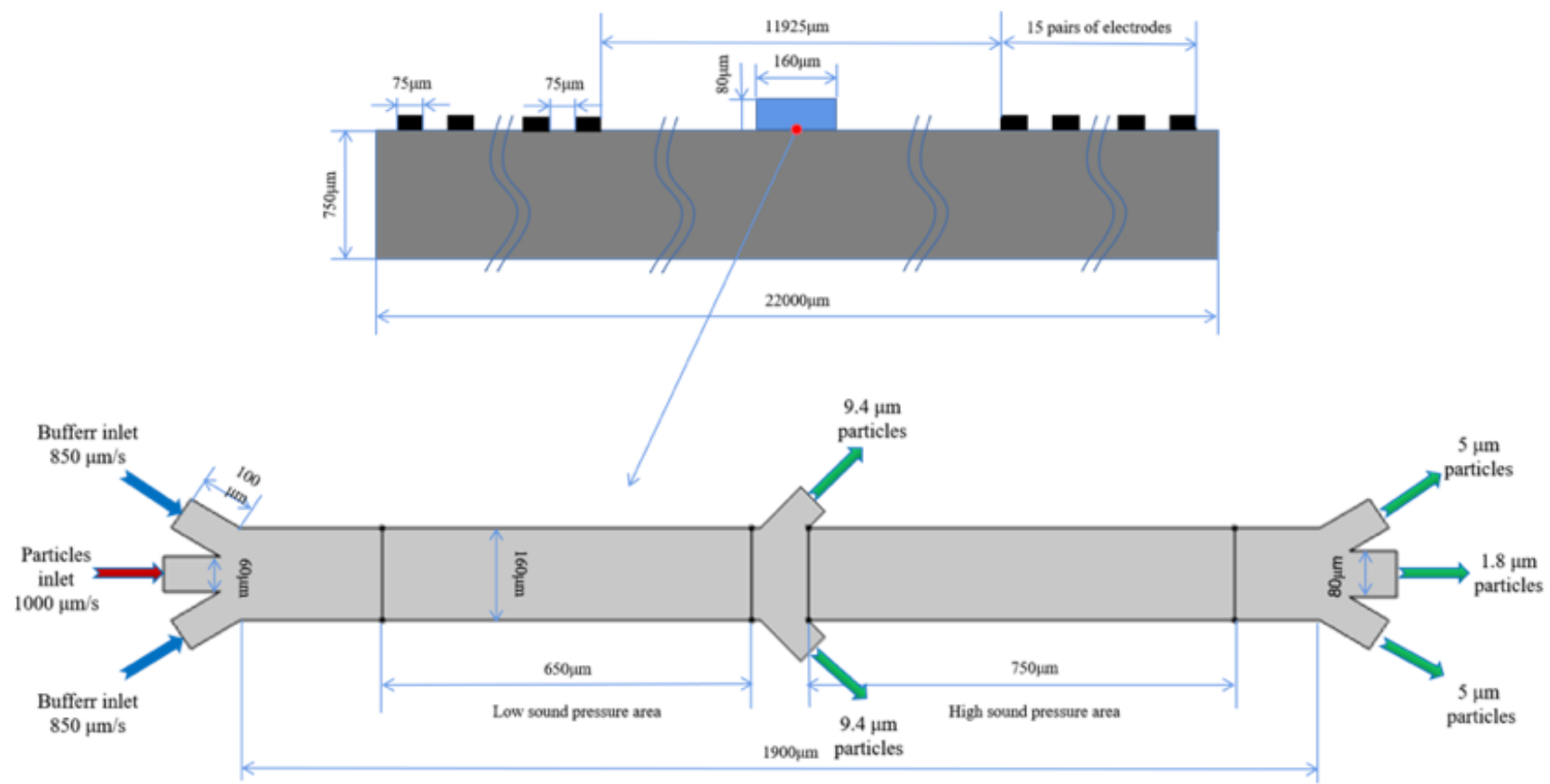

Figure 3

Schematic diagram of cross-section based on SSAW drive system 


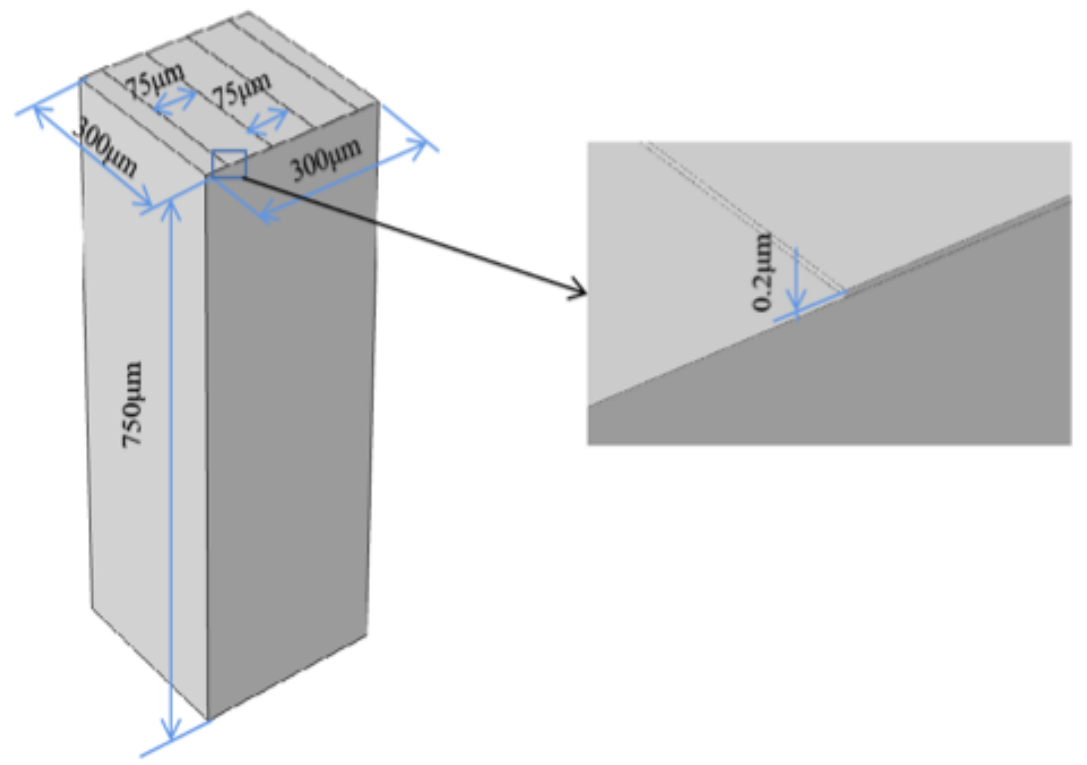

(a)

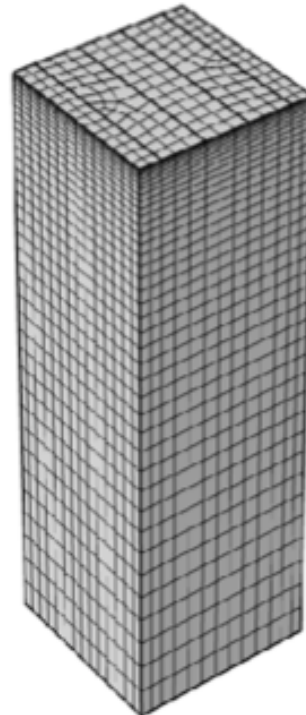

(b)

\section{Figure 4}

The simplified diagram of the three-dimensional model of a surface acoustic wave device. (a) Simplified model (b) Schematic diagram of grid 


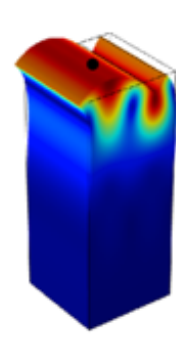

$128^{\circ} \mathrm{YX}-\mathrm{LiNbO} 3$

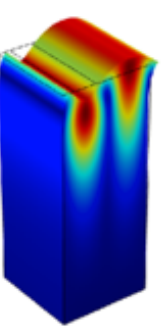

Piezoelectric ceramic PZT-5H
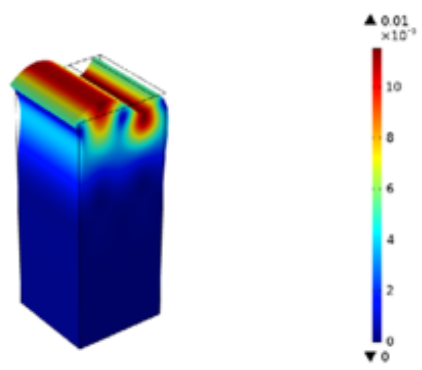

$\mathrm{ZnO}$ single crystal

$4 \underset{\times 10^{-1}}{196 \times 10^{-7}}$

18
16
16
14
12
10
6
6
4
2
70

(a)
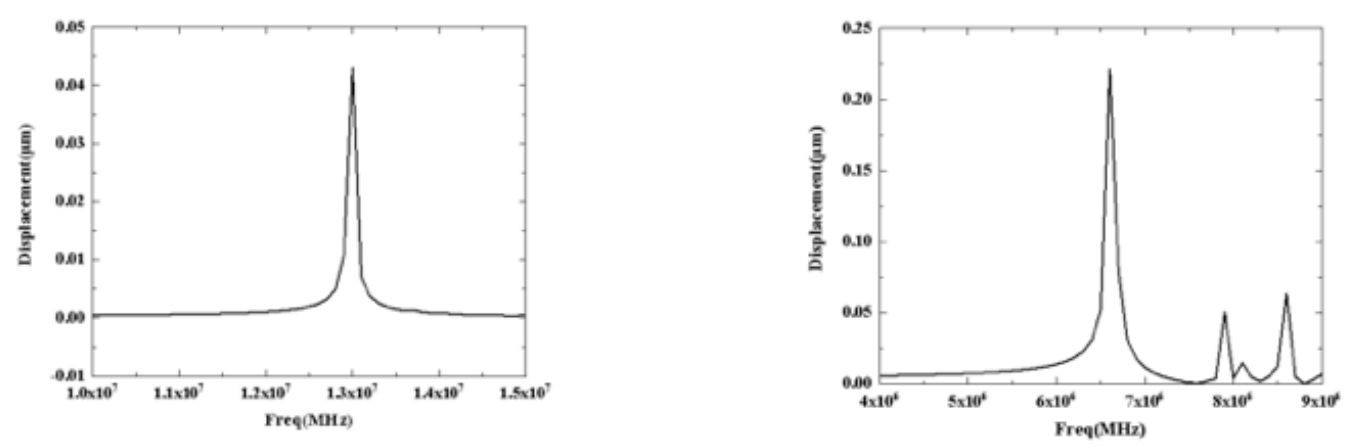

$128^{\circ} \mathrm{YX}-\mathrm{LiNbO3}$

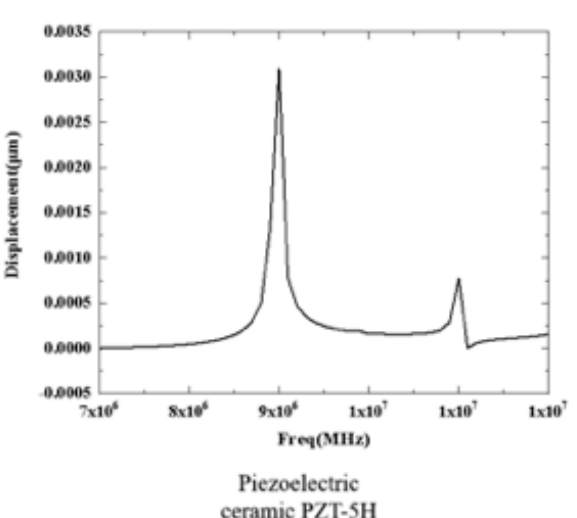

$\mathrm{ZnO}$ single crystal

(b)

\section{Figure 5}

Modal analysis of the model and harmony response analysis. (a) The mode diagram of the model at the resonance frequency. (b) The relationship between the displacement of the center node of the model electrode and the frequency. 


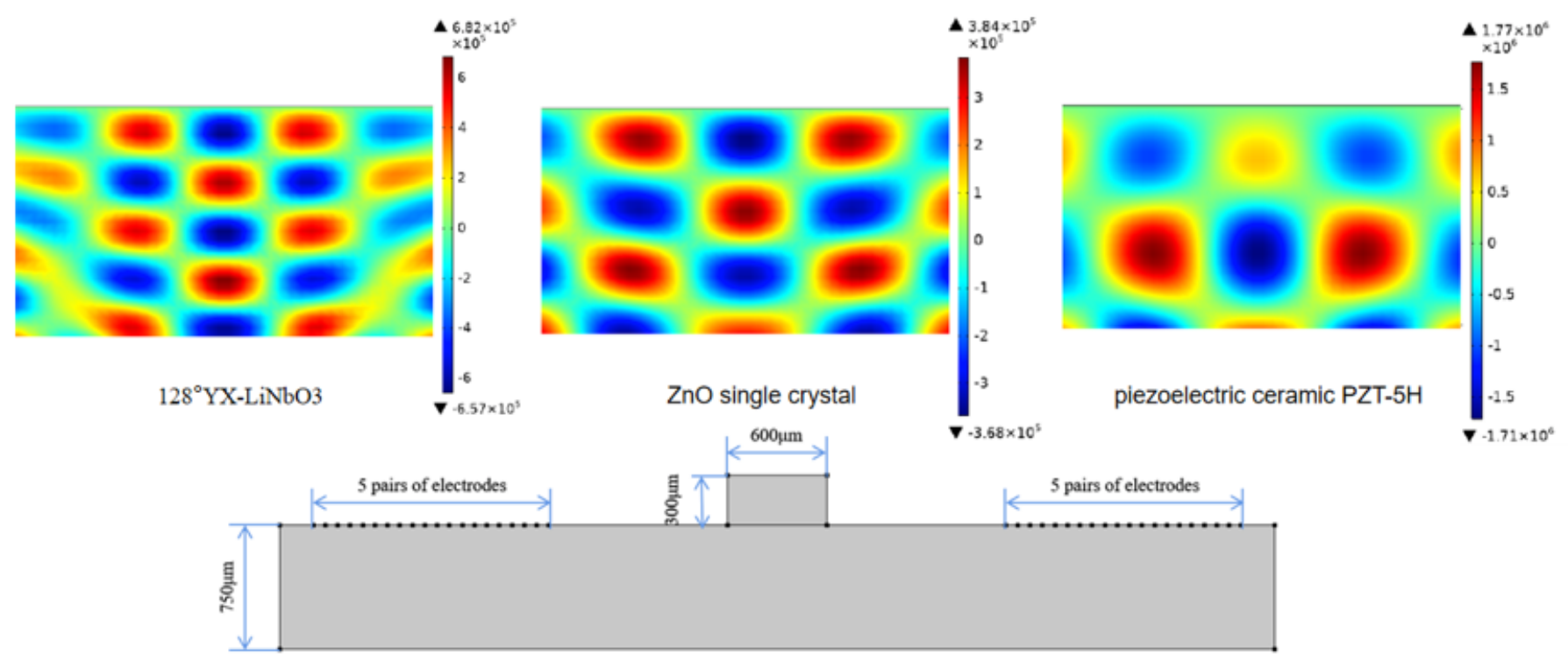

Figure 6

Sound pressure distribution in waters under different base materials

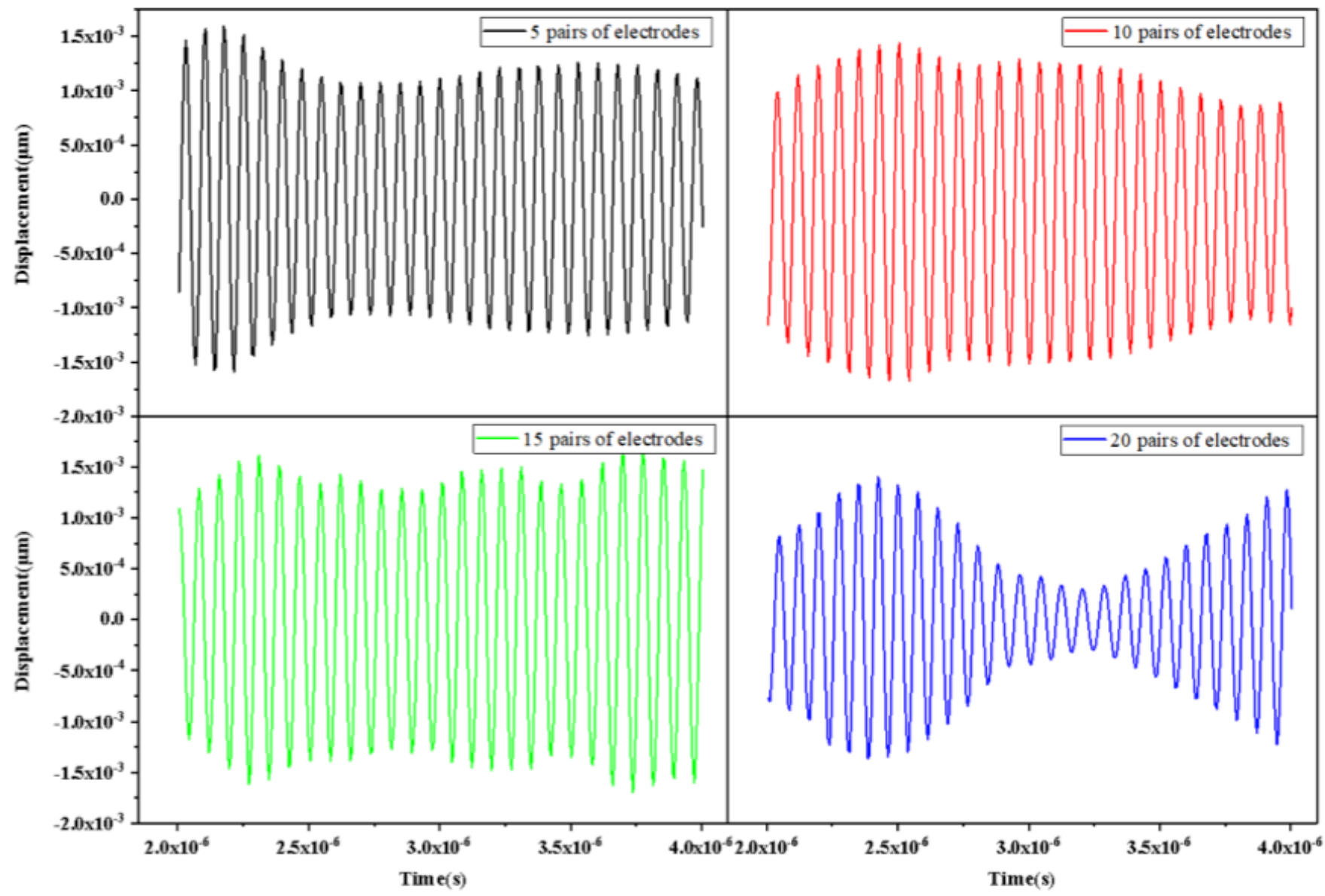

Figure 7 
The output waveform of the observed point in the $Y$ direction

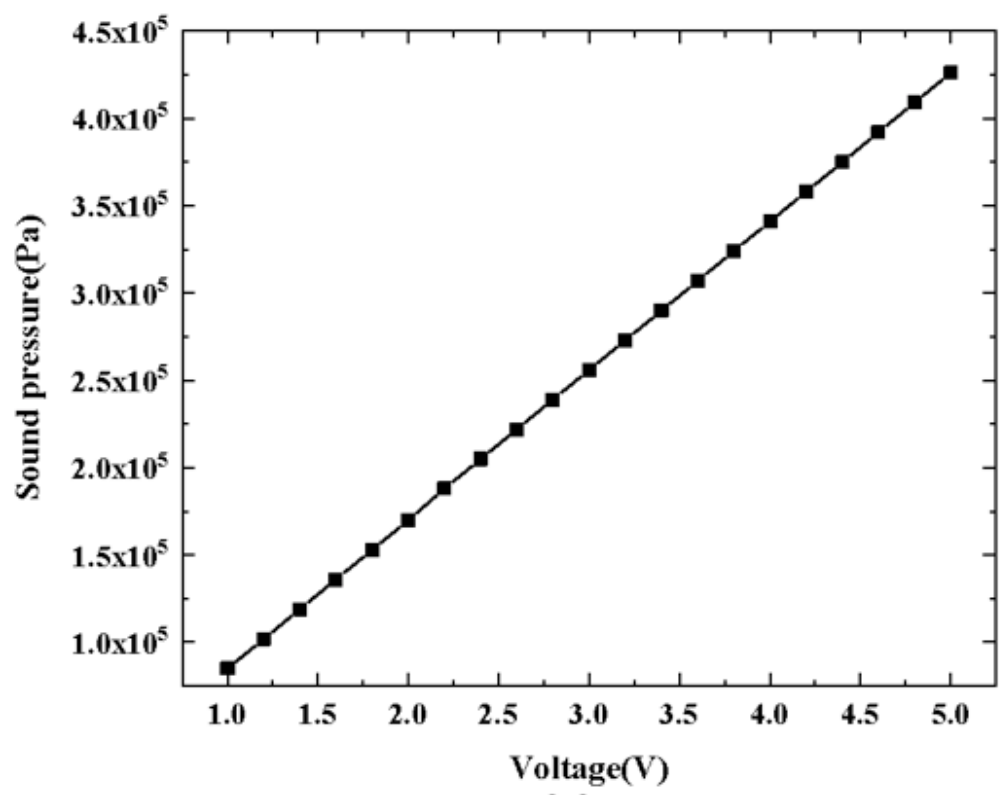

(a)

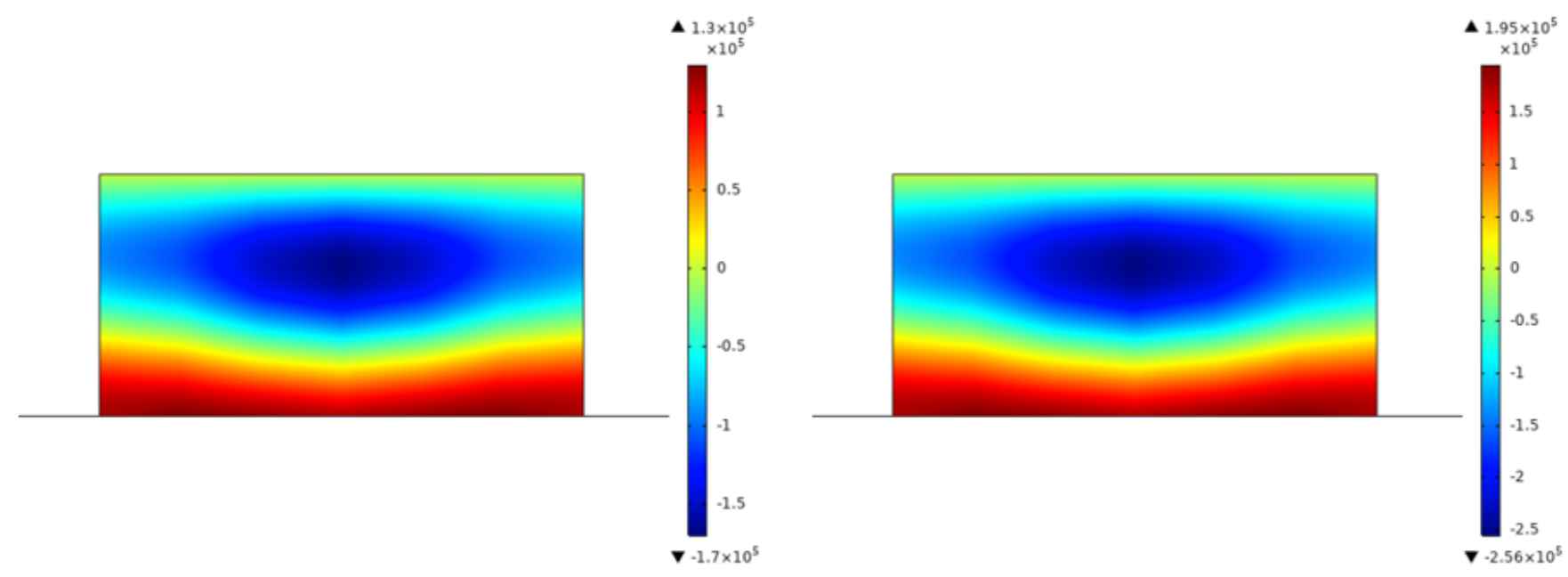

(b)

\section{Figure 8}

Sound pressure in the microchannel liquid (a) The relationship between the transducer electrode voltage and the absolute value of the maximum sound pressure in the liquid. (b) Sound pressure distribution in liquid 


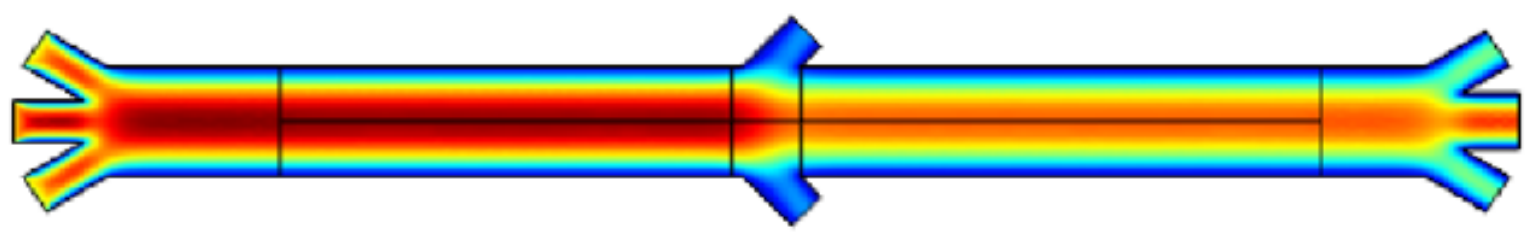

(a)

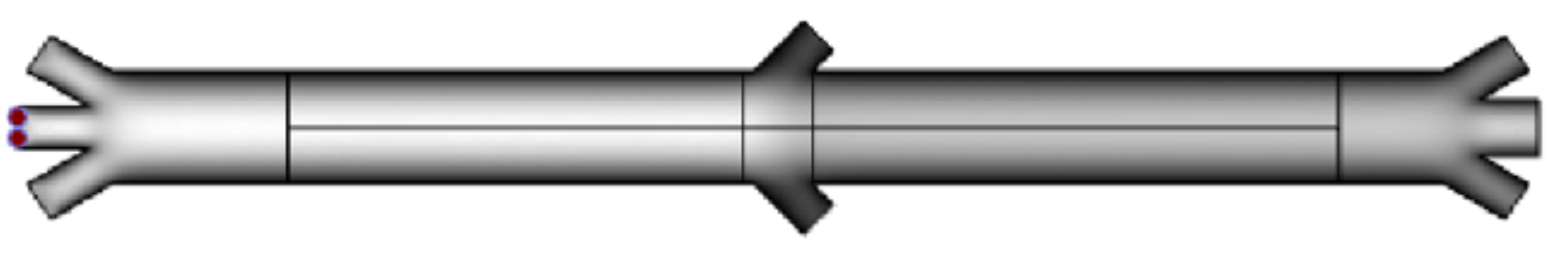

$\mathrm{t}=0 \mathrm{~s}$
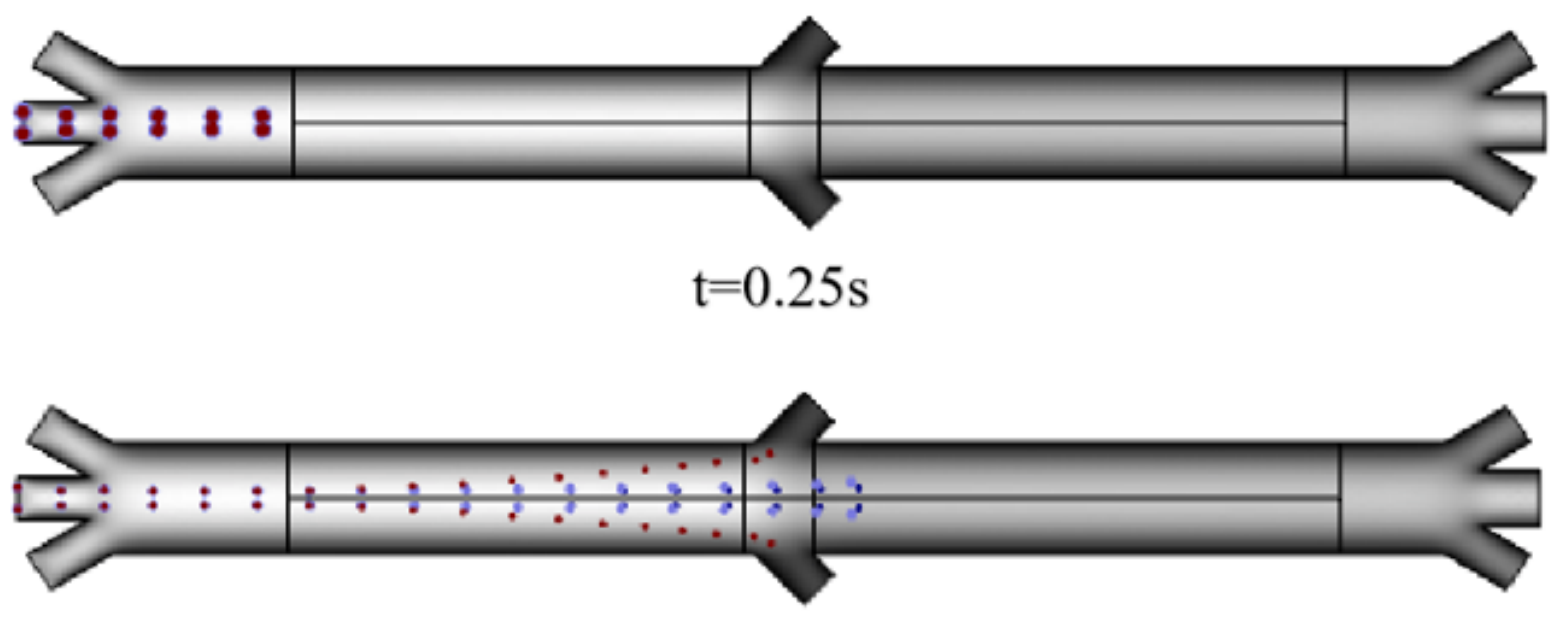

$\mathrm{t}=0.85 \mathrm{~s}$

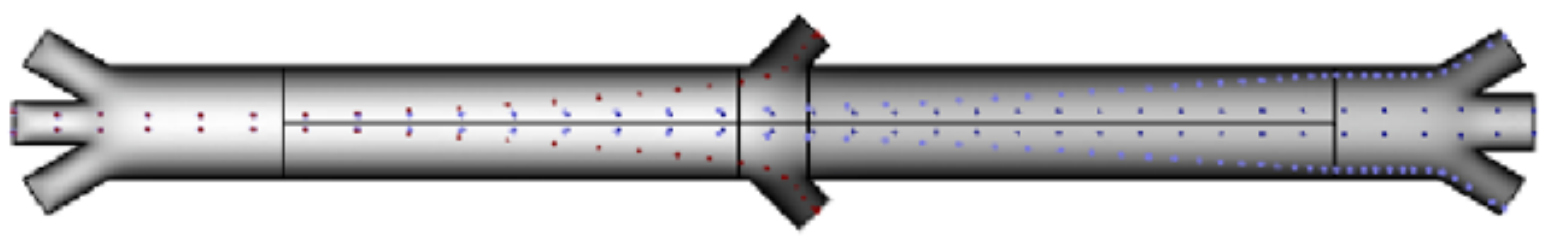

$$
\mathrm{t}=2.4 \mathrm{~s}
$$

(b)

\section{Figure 9}

Flow field and particles in the microchannel. (A) Flow field distribution diagram (b) Particle separation process 